\title{
Technical Developments for MR-Guided Microwave Thermocoagulation Therapy of Liver Tumors
}

\author{
Shigehiro Morikawa ${ }^{1}$, Toshiro Inubushi ${ }^{1}$, Yoshimasa Kurumi ${ }^{2}$, \\ Shigeyuki Naka ${ }^{2}$, Koichiro Sato ${ }^{2}$, Tohru Tani ${ }^{2}$, Nobuhiko Hata $^{3}$, \\ Viswanathan Seshan ${ }^{4}$, and Hasnine A Haque ${ }^{4}$ \\ ${ }^{1}$ Molecular Neuroscience Research Center, ${ }^{2} 1$ st Dept. of Surgery, and \\ ${ }^{3}$ Department of Neurosurgery, Shiga University of Medical Science, Seta Tukinowa-cho, \\ Ohtsu, Shiga 520-2192, Japan, \\ ${ }^{3}$ Graduate School of Information Science and Technology, The University of Tokyo, Hongo 7- \\ 3-1, Bunkyo-ku, Tokyo 113-8656, Japan \\ ${ }^{4}$ GE-Yokogawa Medical Systems, 4-7-127, Asahigaoka, Hino, Tokyo191-8503, Japan.s
}

\begin{abstract}
We have started clinical studies of MR-guided thermocoagulation therapy of liver tumors. Through this therapy for two years, we have developed new instruments, such as a filter to reduce noise in MRI from the microwave coagulator, a new MR-compatible electrode for easy detection of the tip position and an MR-compatible endoscopic system for trans-diaphragmatic approach to liver tumors just below the diaphragm. Concerning software, a program was modified for the real-time display of MR temperature map with a scale bar. A navigation software, 3D Slicer was customized to add real-time image navigation capability. The re-sliced images in the two perpendicular planes complemented the limitations of real-time MR image, which is taken in 2-3 seconds. These technical developments play important roles for more accurate, safer and easier treatment.
\end{abstract}

\section{Introduction}

An open configuration MR system enabled us new minimally invasive surgical techniques under the guidance of MR images [1]. Laser beams [2], radiofrequency ablation [3] and cryosurgery [4], have been utilized as thermo-ablation therapies of various tumors in MR environment. In Japan, microwave coagulators, operating at 2.45 $\mathrm{GHz}$, have been developed in liver surgery for hemostasis and tissue destruction for over two decades $[5,6]$. They have also been used in interventional therapy for liver tumors under ultrasonographic or laparoscopic guidance [7, 8]. Microwave thermocoagulation therapy has already been established as one useful minimally invasive therapy for liver tumors in Japan. Since microwave ablation does not cause electromagnetic interference in MR images [9], MR temperature monitoring during ablation might be possible for real-time evaluation of the therapeutic effects. Therefore, we have started clinical studies of MR-guided microwave thermo-coagulation therapy of liver tumors and have experienced more than 100 cases since January, 2000. Through this therapy for two years, we have developed new MR-compatible surgical instruments and computer-assisted image navigation system for more accurate, safer and 
easier treatment. In the present study, the technical developments for this therapy will be presented.

\section{Methods}

All MR data were collected on a double-donut type 0.5 T SIGNA SP/i system (GE Medical Systems, Milwaukee, WI), (Fig. 1A). In this system, a FlashPoint Model 5000 (Image Guided Technologies Inc., Boulder, CO) was included for image plane control. The surgeon can determine the image plane, which exactly includes the path of needle, with a 3-point hand piece having 3 LEDs (Fig. 1B) and three detectors for the infrared light from them are fixed on the ceiling. Real time MR images of $256 \mathrm{x}$ 128 resolution for fluoroscopy were collected using a spoiled gradient echo (SPGR) with $14 \mathrm{~ms}$ TR, $3.4 \mathrm{~ms}$ TE and $30 \mathrm{~cm}^{2}$ FOV. Flip angle was adjusted at a range of 3070 degrees to obtain good contrast of the target. Temperature mapping data were acquired using SPGR with $50 \mathrm{~ms}$ TR, $12 \mathrm{~ms} \mathrm{TE}$ and $24 \mathrm{~cm}^{2}$ FOV. Temperature changes were calculated by the proton resonance frequency method [10].

A microwave coagulator, Microtaze (Model OT-110M, Azwell, Osaka, Japan), which is operated at $2.45 \mathrm{GHz}$, was used as a heating device (Fig. 2A). A custommade notch filter was inserted in the output line in order to reduce noise during ablation. MR-compatible needle type electrodes (250 $\mathrm{mm}$ long, $1.6 \mathrm{~mm}$ in diameter) was custom-made with brass coated with silver and gold (Fig. 2B). Generally, the procedures were carried out under general anesthesia. Liver tumors were percutaneously punctured by a $15 \mathrm{~cm}$ long 14G MR-compatible biopsy needle (Daum, Schwerin, Germany) under the guidance of real time MRI. The electrode was inserted into the tumor through the outer sheath of the needle. Usually, three 60-second ablations were repeated at the same point. Preliminary studies during laparotomy revealed such ablations caused an oval-shaped coagulated area $20 \mathrm{~mm}$ in diameter and $30 \mathrm{~mm}$ long along the axis of the electrode. Ablations and punctures were repeated depending on the size and number of tumors.

To prepare an MR-compatible endoscopic system, ferromagnetic parts of a CCD camera and C-mounted lens (CN42H and T627R, Elmo, Japan) were replaced to nonmagnetic ones and combined with a $220 \mathrm{~mm}$ long non-magnetic telescope (K7210AWA, Storz, Germany). This endoscopic system was used as a thoracoscope for the percutaneous and trans-diaphragmatic puncture of liver tumors just below the diaphragm. The endoscopic image was combined with MR images using the picturein-picture function of a video mixer (MX-1, Futek, Tokyo, Japan).

A navigation software, 3D Slicer [11, 12], which was developed in Brigham \& Women's Hospital, Boston, MA, was installed in an independent SUN Ultra 60 workstation and connected with the MR system by network. High resolution 3D MR volume data were acquired just before the surgical procedures and were registered in the 3D Slicer. The display of the work station was converted to NTSC signal by a downconverter (DC 65A, Imagenics, Tokyo, Japan) and sent to the surgeons through a video switcher of the MR system. 


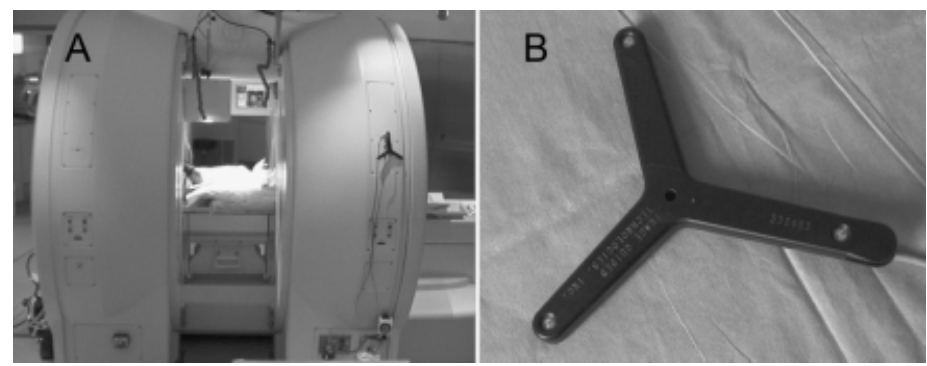

Fig. 1. (A) A double-donut type 0.5 T SIGNA SP/i system (GE Medical Systems, Milwaukee, WI) and a hand piece for image plane navigation system, FlashPoint Model 5000 (Image Guided Technologies Inc., Boulder, CO)

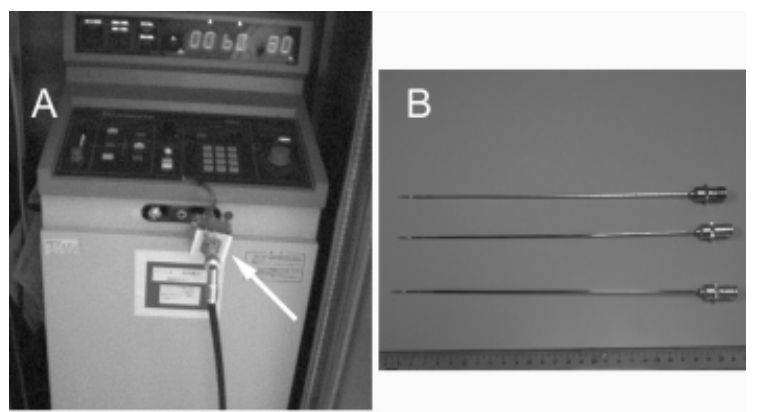

Fig. 2. (A) A microwave coagulator, Microtaze (Model OT-110M, Azwell, Osaka, Japan). A custom-made notch filter was inserted in the output line (arrow). (B) MR-compatible needle type electrodes

\section{Results and Discussion}

\section{Instruments for Microwave Ablation}

Initially, as shown in Fig. 3A, electromagnetic interference was appeared during microwave irradiation. A notch filter, which attenuates $40 \mathrm{~dB}$ at $21.25 \mathrm{MHz}\left({ }^{1} \mathrm{H}\right.$ resonance frequency at $0.5 \mathrm{~T}$ ) and $0.3 \mathrm{~dB}$ at $2.45 \mathrm{GHz}$, was inserted in the output line (Fig. 2A, arrow). After the installation of the filter, the noise in MRI disappeared even during microwave ablation (Fig. 3B).

For the microwave ablation, the electrode tip should be protruded at least by $2 \mathrm{~cm}$ from the needle. The $14 \mathrm{G}$ needle was easily detected in real time MRI, but the electrode tip was not always visible, because its susceptibility effect was only slight. It was advantageous for the data collection of temperature mapping. The length of the protrusion was controlled using a plastic tube as a stopper. However, in some cases, accurate detection of the electrode tip position was required. For such cases, another type of "firefly" electrode, which contained a small stainless steel part in the tip (Fig. 4A). The signal defect exactly corresponded to the tip position under our real- 
time MR imaging condition. It could not be used for temperature data acquisition, but enabled safer and more accurate puncture of the tumors near the large vessels (Fig. 4 $\mathrm{B}$ and $\mathrm{C})$.
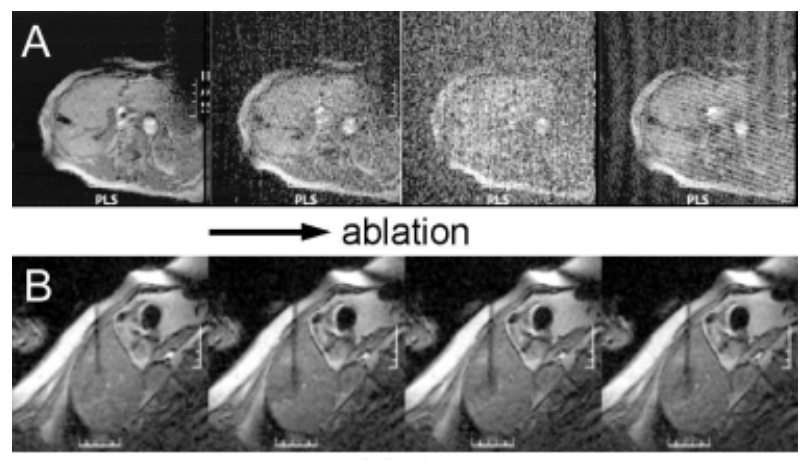

ablation

Fig. 3. Real time MR images of the liver without (A) and with (B) a notch filter
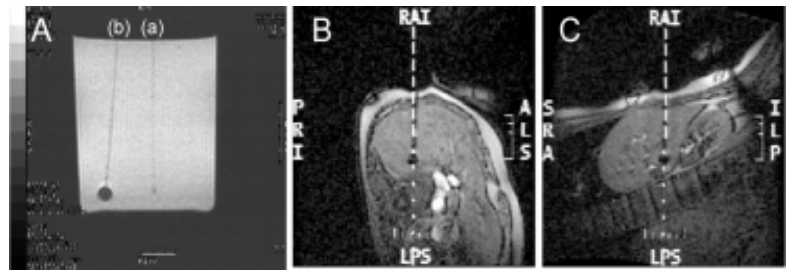

Fig. 4. (A) An MR image of standard (a) and "firefly" (b) type electrodes in an agar phantom. (B) Axial and (C) sagittal view of liver during puncture for a tumor near the inferior vena cava using the "firefly" type electrode

\section{MR-Compatible Endoscopic System}

For the treatment of the tumor located just below the diaphragm, the percutaneous puncture from the abdominal wall is not easy. As shown in Fig. 5, the route of the needle is long and might injure vessels or bile ducts. For such a tumor, transdiaphragmatic approach is much easier and safer. To realize this approach, an MRcompatible thoracoscope was combined with MR-guidance. Both MR images and endoscopic image were sent to the surgeon using the picture-in-picture function (Fig. 6). Main and accessory images were exchanged depending on the surgeon's request. In this approach, the right lung is collapsed under general anesthesia using a double-lumen endotracheal tube. The microwave ablation can be carried out sufficiently without taking care of the injury of the lung caused by heating. Thoracoscope could also detect bleeding from the diaphragm or chest wall, which could be easily stopped by the microwave. The combination of surface information by endoscope and in depth by MRI was found to be very useful for increasing the safety, reliability and availability of this procedure. 


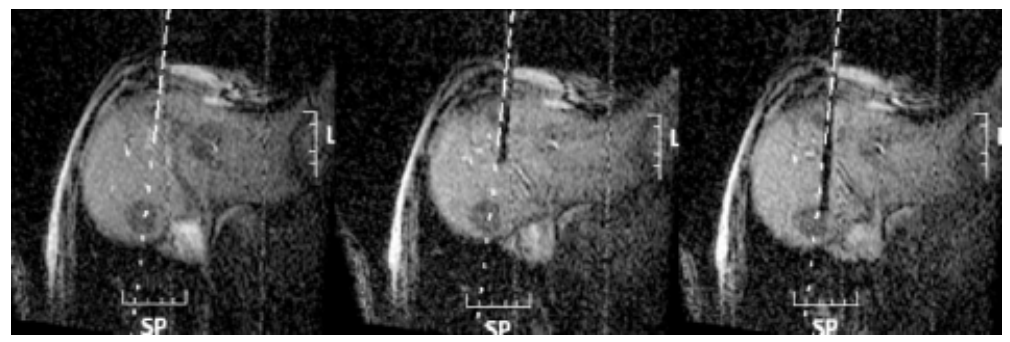

Fig. 5. Real time MR images during the puncture of a liver tumor located just below the diaphragm from the abdominal wall
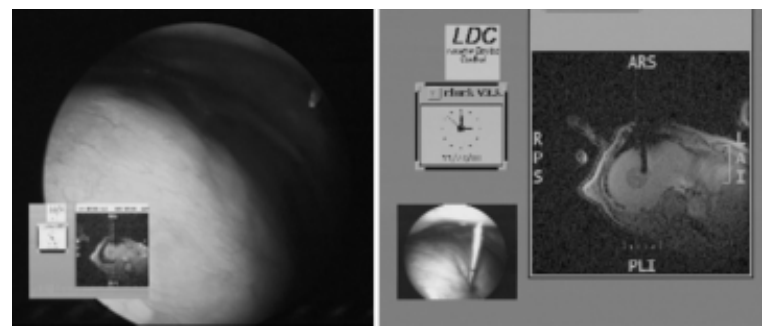

Fig. 6. Combined display of real time MR and thoracoscopic images for the transdiaphragmatic puncture of the liver tumor

\section{MR Temperature Monitoring}

Initially, MR temperature monitoring during microwave ablation was impossible because of the severe noise. After the installation of the filter, good temperature maps could be obtained with agar phantom and beef liver (Fig. 7). The software was modified for real time color display of the temperature map with a scale. However, in the clinical cases, temperature monitoring was not easy. The most serious factor is the movement of the liver. Movement of surgeons and surgical instruments in the operating field also affected the magnetic field and the results of temperature calculation. MR temperature data during microwave ablation were collected while suspending the artificial ventilation (Fig. 8). In the practical aspect, it takes some time to change the acquisition parameters from real time MRI to temperature map, which also makes the temperature monitoring difficult. At present, temperature monitoring of every ablation in the procedure is impossible. In order to utilize MR temperature map for the evaluation of therapeutic effects of this procedure, further developments are required. A dynamic study using contrast media at the end of the procedure is utilized for the evaluation.

\section{Customization and Utilization of a Navigation Software, 3D Slicer}

The 3D Slicer, which we got from Brigham \& Women's Hospital, did not support the real time image navigation capability. A program was prepared in SIGNA SP system to transfer the information of the hand piece position and real-time MR images to the 3D Slicer continuously. The 3D Slicer was customized to display the transferred realtime MR image and two re-sliced images (same and perpendicular planes to the real- 
time image) from the 3D volume data registered beforehand (Fig. 9). In other words, all the 3 images were controlled by the surgeon using the hand piece. The image resolution and quality of real time MR images were not necessarily satisfactory because only a limited time of 2-3 seconds was allowed for their data acquisition. The two re-sliced images showed better image quality and faster response to the hand piece. The images in the two perpendicular planes made the recognition of the target position easy. Of course, the needle position must be recognized on the real time image, but the re-sliced images complemented the limitations of real-time MR image. These combinations enabled us to accomplish more accurate and safer image navigation of the liver. The tumor in Fig. 9 (white arrow), which located near the hepatic vein and the inferior vena cava, was successfully punctured without injuring the vessels. The information of the hand piece status (visible or blocked) is very important, because the needle might possibly be dislocated from the target. To inform this status to the surgeon, a button was added in the menu bar (Fig. 9, black arrow). Besides them, flip function of each image display for the convenience of the procedure and color display with a scale bar for real-time temperature map were added to the $3 \mathrm{D}$ slicer. In the case with thoracoscope, endoscopic image was combined to the 3D Slicer and sent to the surgeon (Fig. 10). Now, this software is used routinely in this procedure.

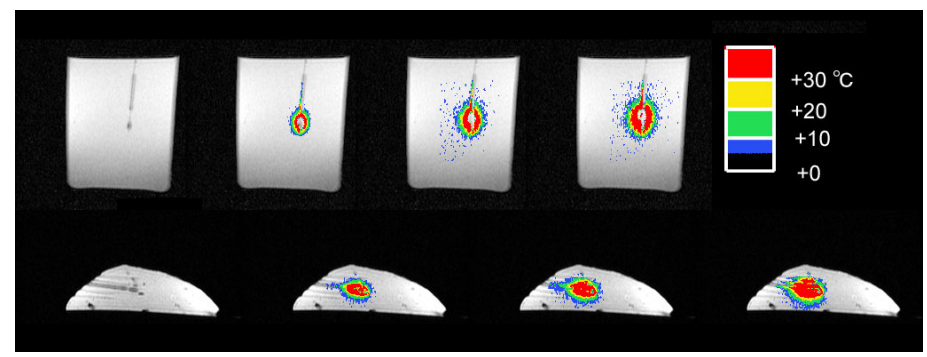

Fig. 7. Temperature maps of an agar phantom (upper) and beef liver (lower) during microwave ablation at $0,1,2$, and 3 minutes

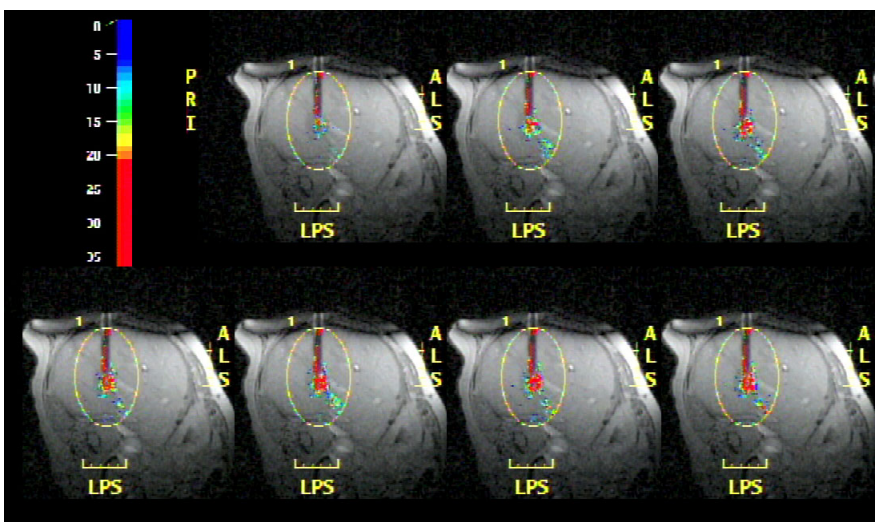

Fig. 8. Temperature maps of a liver tumor during 1-min microwave thermo-coagulation therapy 


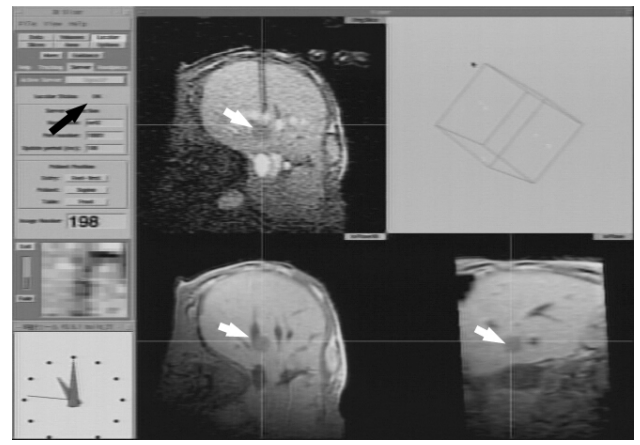

Fig. 9. Real time image navigation for microwave thermocoagulation therapy of a liver tumor using the 3D Slicer. The upper left is a real time MR image and the lower two are re-sliced images (same and perpendicular planes to the real-time image) from the $3 \mathrm{D}$ volume data. The tumor is indicated with white arrows. The hand piece status (visible or blocked) is displayed in the menu bar (black arrow)

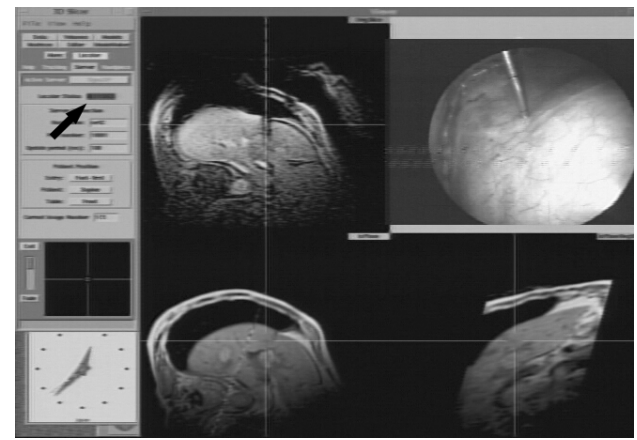

Fig. 10. Real time image navigation for thoracoscope assisted microwave thermo-coagulation therapy of a liver tumor using the 3D Slicer. The upper right is a endoscopic image showing a needle through the diaphragm. The arrangements of other windows are the same as Fig. 9. The button (black arrow) indicates the hand piece is blocked with red color

\section{Conclusions}

For the MR-guided thermo-coagulation therapy of liver tumors, we have developed new instruments such as the filter, electrode and endoscope, and software for temperature monitoring and image navigation. These technical developments play important roles for more accurate, safer and easier treatment.

\section{Acknowledgments}

We are indebted to Dr. F. Jolesz and Dr. R. Kikinis (Brigham \& Women's Hospital) for donating the 3D Slicer. 


\section{Reference}

1. Schenck, J.F., Jolesz, F.A., Roeme,r P.B., et al.: Superconducting open-configuration MR imaging system for image-guided therapy. Radiology 195 (1995) 805-814.

2. Schulze, C.P., Kahn, T., Harth, T., Schwurzmaier, H.-J., Schober, R.: Correlation of neuropathologic findings and phase-based MRI temperature maps in experimental laserinduced interstitial thermotherapy. J. Magn. Reson. Imaging 8 (1998) 115-120.

3. Lewin, J.S., Connell, C.F., Duerk JL, et al.: Interactive MRI-guided radiofrequency interstitial thermal ablation of abdominal tumors: clinical trial for evaluation of safety and feasibility. J Magn Reson Imaging 8 (1998) 40-47.

4. Tacke, J., Adam, G., Haage, P., Sellhaus, B., Grosskortenhaus, S., Gunther, R.W.: MRguided percutaneous cryotherapy of the liver: in vivo evaluation with histologic correlation in an animal model. J. Magn. Reson. Imaging. 13(2001) 50-56.

5. Tabuse, K.: A new operative procedure of hepatic surgery using a microwave tissue coagulator. Arch. Jpn. Chir. 48 (1979) 160-172.

6. Hamazoe, R., Hirooka, Y., Ohtani, S., Kato, T., Kaibara, N.: Intraoperative tissue coagulation as treatment for patients with nonresectable hepatocellular carcinoma Cancer 75 (1995) 794-800.

7. Seki, T., Wakabayashi, M., Nakagawa, T., et al.: Ultrasonically guided percutaneus microwave coagulation therapy for small hepatocellular carcinoma. Cancer 74 (1994) 817825.

8. Ido, K., Isoda, N., Kawamoto, C., et al.: Laparoscopic microwave coagulation for solitary hepatocellular carcinoma performed under laparoscopic ultrasonography. Gastrointest. Endosc., 45 (1997) 415-420.

9. Chen, J.C., Moriarty, J.A., Derbyshire, J.A., et al.: Prostate cancer: MR imaging and thermometry during microwave thermal ablation-Initial experience. Radiology 214 (2000) 290-297.

10. Ishihara, Y., Calderon, A., Watanabe, H., et al.: A precise and fast temperature mapping using water proton chemical shift. Magn. Reson. Med. 34 (1995) 814-823

11. Jolesz, F.A., Nabavi, A., Kikinis, R.: Integration of interventional MRI with computerassisted surgery. Journal of Magnetic Resonance Imaging. 13 (2001) 69-77

12. Gering, D.T. Nabavi, A., Kikinis, et al.: An integrated visualization system for surgical planning and guidance using image fusion and an open MR. Journal of Magnetic Resonance Imaging. 13 (2001) 967-975. 